\title{
Corela
}

Cognition, représentation, langage

HS-19 | 2016

Le point de vue pris au mot

\section{Marquage ou contrainte ? Regard heuristique sur les usages du concept de point de vue}

\section{Zsuzsa Simonffy}

\section{(2) OpenEdition}

\section{Journals}

\section{Édition électronique}

URL : http://journals.openedition.org/corela/4281

DOI : $10.4000 /$ corela.4281

ISSN : $1638-573 \mathrm{X}$

\section{Éditeur}

Cercle linguistique du Centre et de I'Ouest - CerLICO

\section{Référence électronique}

Zsuzsa Simonffy, "Marquage ou contrainte ? Regard heuristique sur les usages du concept de point de vue », Corela [En ligne], HS-19 | 2016, mis en ligne le 08 juin 2016, consulté le 21 avril 2019. URL http://journals.openedition.org/corela/4281; DOI : 10.4000/corela.4281

Ce document a été généré automatiquement le 21 avril 2019

\section{(c) (i) (2)(2)}

Corela - cognition, représentation, langage est mis à disposition selon les termes de la licence Creative Commons Attribution - Pas d'Utilisation Commerciale - Partage dans les Mêmes Conditions 4.0 International. 


\title{
Marquage ou contrainte? Regard heuristique sur les usages du concept de point de vue
}

\author{
Zsuzsa Simonffy
}

La vraie question n'est pas de savoir si le contact d'un bec de pic guérit les maux de dents, mais s'il est possible, d'un certain point de vue, de faire

"aller ensemble" le bec de pic et la dent de l'homme. (Lévi-Strauss 1962 : 21)

L'homme parlant parle l'écoute qu'il imagine à sa propre parole (Barthes $1978: 10$ )

\section{Préambule}

1 Il est indéniable que les travaux de Pierre-Yves Raccah, depuis le début de sa carrière scientifique dans le domaine de la linguistique théorique, fournissent une assise intellectuelle nouvelle à tous ceux qui se méfient de l'illusion représentationnaliste du sens. Dans le cadre de notre contribution, nous ne pouvons pas prendre en considération son système de pensée dans toute son ampleur. Nous nous limiterons à mettre en évidence la place qui est la sienne dans la formation du concept de point de vue car, si d'autres auteurs ont ponctuellement employé ce concept dans un sens générique aussi bien que dans un sens spécifique, le relief qu'il lui a donné fut décisif à l'évolution interne de la sémantique. Chacun l'a interprété dans un sens propre ou l'a utilisé dans ses variantes telles que perspective, focalisation, empathie, camera angle avant tout comme un outil de décentrement du sujet, mais c'est Pierre-Yves Raccah qui l'a installé, dans la perspective d'une discipline, antérieur à toute détermination cognitive ou phénoménologique. Il voit dans le point de vue un dispositif scientifique permettant une avancée à la fois théorique et descriptive en la recherche de possibles réponses à un 
grand nombre d'interrogations concernant la langue, la signification des unités linguistiques et leurs effets de sens.

Toute information sur notre monde est filtrée par nos sens est biaisée par les valeurs issues de notre culture, circulant dans les différents genres de discours au sein de la société. Malgré cela, nous savons que le monde réel existe et que nous devons choisir les théories qui rendent comptent des phénomènes observés pour les expliquer le mieux possible. Comment y procéder? La Sémantique des Points de Vue nous en esquisse un chemin à suivre.

3 L'étude à laquelle nous nous livrons dans l'objectif de déceler des aspects insoupçonnés de l'usage du point de vue - qui relève le plus souvent des registres de la narratologie ou de l'épistémologie -, n'est pas, par ailleurs, sans antécédents ni dans la littérature linguistique, ni dans nos travaux. Cependant, objecter que le concept de point de vue n'est pas une invention récente, mais, dans une visée épistémologique, il est marqué de paradigme saussurien ${ }^{1}$ et, de plus, qu'il a été largement étudié, ne va rien changer à l'originalité du modèle qu'il est destiné à fonder. Si nous reprenons le fil de la réflexion à cette occasion de rendre hommage à Pierre-Yves Raccah, c'est pour mettre en valeur, entre héritage et réappropriation, son apport à la définition sémantique du concept en tenant compte de ce qui retarde et favorise l'émergence de ce concept en sémantique, dans ses rapports avec les travaux qui répondent, par ailleurs, à des intérêts d'ordre différent notamment cognitif, culturel ou social.

\section{Introduction : confusion entre usage courant et usage savant}

4 Ce que le terme de point de vue désigne est fluctuant et imprécis, et inversement, le même concept trouve des dénominations bien variées. Il est intéressant à noter que :

[...] dans la langue française «classique » jusqu'au XVIII siècle disons, l'expression « point de vue » désignait aussi, et très logiquement, l'endroit où l'on doit placer un objet pour le rendre mieux visible. Admirable ambiguïté de la langue, qui entérinait la fondamentale dualité regardant / regardé. (Aumont $1983: 3$ )

5 Pour faire l'état des lieux sur les usages du concept de point de vue dans le domaine des sciences humaines, nous allons procéder de manière un peu inhabituelle. Comme nous ne pratiquons pas nos recherches dans un esprit historique, ce serait fortuit de faire un effort pour en retracer une évolution suivant un ordre chronologique strictement établi.

Cependant, sans prétendre d'approfondir une analyse étymologique et dictionnairique ${ }^{2}$ ou de procéder à la restauration du passé, il nous convient d'en rappeler une considération d'ordre généalogique. Le point de vue apparaît à l'origine dans son sens optique comme un concept dynamique dans la mesure où il renvoyait à l'œil, plus précisément au mouvement oculaire. C'est seulement plus tard qu'il a pris, dans le domaine pictural avec le triomphe de la représentation en perspective suscitant les effets d'éloignement, une acception qui met en parenthèse tout aspect du mouvement oculaire. Il nous semble que, contrairement à cette dynamique, il est aussi susceptible de délimiter l'objet même de tout autre, la vue arrêtée à ses limites ne pouvant s'étendre au-delà de l'espace borné. Cette capacité à clôturer ou à dessiner les contours donne l'impression de la fixation et de l'isolement de l'objet de tout ce qui ne le constitue pas, pareil à un encadrement, dans ses limites restreintes. 
Ceux qui s'intéressent aux acceptions courantes, doivent être confrontés donc au fait que le concept de point de vue se forge dans les arts visuels pour se transposer plus tardivement dans le langage courant. Dans la perspective de cette transposition, si le langage courant lui donne un sens étendu, c'est dû certainement aux associations avec les catégories esthétiques des arts visuels telles que perspective, vue, - et avec l'apparition du cinéma -, image, caméra. L'usage de ces catégories indépendamment de leur fonction à remplir dans un système esthétique établi, nous rappelle un point pertinent pour notre propos, à savoir, le fait que la perception ne pourrait pas être réduite au visuel tout court. De surcroit, la vue qui est d'ordre sensoriel s'apparente à la vision faisant référence à la façon dont le monde est perçu à travers la pensée, qui, elle, est difficile à saisir sans aucune détermination discursive ou linguistique.

Pour illustrer cette ambiguïté du concept de point de vue tout en restant sur le plan visuel, plus précisément le glissement de la vue vers la vision, nous recourons à un usage apparemment épisodique mais qui sous-tend réellement d'autres usages. Dans son ouvrage traitant quelques problèmes linguistiques, Calvet (2004:21-30) répond par une analogie aux interrogations concernant le caractère scientifique des recherches ${ }^{3}$. Sans revenir sur les enseignements de son expérience de pensée y compris le rôle de l'analogie dans le discours scientifique, - ce qui nécessiterait des développements trop longs pour être intégrés dans la présente étude -, tournons plutôt à ce que nous apprend, pour ce qui nous préoccupe, cette analyse, sur les usages du concept de point de vue. Nous nous contentons de rappeler son constat selon lequel les descriptions produisent un artefact qui change de visage suivant le changement de point de vue sur la base duquel la description est réalisée. Parmi les artefacts générés par les descriptions aucun ne correspond à la réalité. Par extension, c'est valable pour la langue dans le domaine des sciences du langage. Comme il le déclare :

Les procédures de description produisent un artefact, et les différents points de vue linguistiques produisent des langues différentes, dont aucune ne correspond à une quelconque réalité : la langue n'existe pas, elle est une invention des linguistes. Calvet $(2004: 21)$

Dans la perspective de notre analyse conceptuelle du point de vue, c'est la disposition analogue qui retient notre attention. La Montagne Sainte-Victoire a fait l'objet de nombreuses peintures et aquarelles de Cézanne. Plus de quatre-vingts toiles nous témoignent du fait que, chaque fois, le point de vue du peintre nous en donne à voir, de préférence, la façade ouest de la montage, par ailleurs prise sous différents angles. L'artiste, regardant dans la même direction insiste à la représenter dans cette orientation comme un cône tronqué ou inachevé.

Ce que cette analogie nous suggère, c'est que peindre une montagne et décrire une langue reviennent au même dans la mesure où, dans les deux cas, l'objet visé tend à perdre sa complexité; c'est la raison pour laquelle on peut dire respectivement voilà le cône cézanien et voilà l'invention du linguiste. Peu importe que le critère soit tantôt l'angle, tantôt le trait pertinent : il s'agit d'une réduction de même nature, ce qui n'empêche pas, par ailleurs, une approche exhaustive. Il suffit de rappeler que le concept étant relatif à la dimension spatiale - les quatre points cardinaux ou les coordonnées verticale et horizontale, - si l'on envisage un objet sur l'axe longitudinal, il n'y aucune garantie que la vue de dessus permette de conclure sur le même objet que celle de dessous. En somme, le point de vue n'a d'autre fonction que de réduire la totalité à ses parties, ce pouvoir réducteur produisant un trait distinctif ou pertinent. 
11 Qu'est-ce que cette situation anecdotique trahit du concept ? Il en révèle sa polyvalence et nous fournit l'occasion de mettre en évidence ses enjeux. Nous pouvons en dégager trois sens différents qui s'entremêlent bien souvent tout comme dans le cas cité :

- le pdv renvoie à l'emplacement du regardant ;

- le pdv désigne une opinion;

- le pdv indique une façon de considérer quelque chose.

12 Ces trois acceptions correspondent exactement à celles qu'Aumont a relevées pour préciser ce qu'on entend par point de vue dans le domaine des études cinématographiques.

Premièrement, «Le point de vue peut désigner : un emplacement, réel ou imaginaire, depuis lequel une scène est regardée " (1990: 118). En effet, si cet emplacement détermine l'angle qui oriente le regard de Cézanne pour se poser sur la montagne, alors dans son sens optique, il est indiscernable de l'angle. À savoir, si l'artiste a changé son emplacement dans l'espace physique, ces nouvelles positions n'ont rien changé à son orientation vers l'ouest; c'est ainsi que l'objet de son regard, resté dans son angle de vue, ne subit non plus de changement.

Deuxièmement, le point de vue désigne «la façon particulière dont une question peut être considérée » (Aumont 1990 : 118); dans cette même lignée de pensée, nous pouvons ajouter ceci : puisqu'une question peut être envisagée sous différentes angles, recelant de nombreuses facettes, les différentes approches n'aboutissent pas forcément aux mêmes résultats. Probablement, pour Cézanne, la question porte sur les principes picturaux parmi lesquels, en premier lieu, celui du choix du mode de représentation de la montagne. Il privilégie un seul des points cardinaux, à savoir, l'orientation vers l'ouest. S'il avait testé les autres, ses tableaux montreraient autre chose que la forme répétée d'un cône inachevé.

Troisièmement, le point de vue renvoie à « une opinion, un sentiment à propos d'un phénomène ou d'un événement » (Aumont 1990 : 118). Suivant cette acception, le cône cézanien exprime ainsi son opinion ou intuition esthétique à propos du phénomène naturel qui est, en l'occurrence, la Montagne Sainte-Victoire.

Déjà, indépendamment de ladite analogie et de la montagne de Cézanne, il convient de noter que ces trois acceptions dans le domaine de la théorie du cinéma sont recouvertes par le seul terme de cadrage. En effet, la première met en jeu le regard incarné, alors que les deux autres relèvent la vue subjective ou l'image focalisée, selon laquelle la représentation est déjà le produit d'une sélection: les plans indiquent que certains aspects sont pertinents, alors que d'autres sont effacés. Voilà comment le cadrage les englobe, de manière amalgamée :

Le premier de ces sens correspond à ce que nous venons d'exposer, l'incarnation d'un regard dans le cadrage - la question restant à savoir à qui attribuer ce regard, cette vue : au producteur de l'image, à l'appareil, ou dans les formes narratives de l'image (spécialement au cinéma), parfois à une construction déjà imaginaire, à un personnage. Mais, il est en outre intéressant d'observer que les deux autres sens correspondent à d'autres valeurs, connotatives, du cadrage : à tout ce qui, dans les images narratives, lui fait traduire une vision subjective, „focalisée”; plus largement, à tout ce qui est représenté, en le valorisant, en le dévalorisant, en attirant l'attention sur un détail au premier plan, etc. (Aumont 1990 : 118) 


\section{Usages techniques du point de vue}

17 Remarquons que la notion de point de vue proposée par Aumont fait entrer dans sa définition la notion de focalisation ${ }^{4}$ relevant de la narratologie ${ }^{5}$. La raison pour laquelle nous ne voyons pas l'intérêt d'ouvir une section pour traiter à part son usage en études cinématographiques ${ }^{6}$, c'est qu'il est présupposé que l'image possède une structure narrative. Or, la coïncidence entre point de vue et focalisation n'est pas toujours anodine. Il nous faut reconnaître qu'il n'est pas facile de tracer une frontière entre les phénomènes d'ordre narratologique de ceux qui sont d'ordre linguistique ${ }^{7}$, malgré le fait que la portée de la focalisation en narratologie soit le texte et en linguistique, l'énoncé. Si, dans la suite de nos réflexions, nous insisterons à déceler les recoupements prêtant à confusion, c'est parce qu'ils permettent d'anticiper sur les questions dont le traitement constitue un préalable à sa définition sémantique. Par ailleurs, dans la théorie de l'énonciation de la Scapoline, ces recoupements sont nettement assumés par la fusion de la notion de polyphonie héritée de la théorie littéraire et de celle qui est héritée de la linguistique : ils ont pour objectif d'assurer un pont entre les analyses effectuées sur le plan de l'énoncé et celles qui s'imposent sur le plan du texte littéraire, cette extension lui donnant une force toute puissante.

\subsection{Focalisation : narratologie et linguistique}

Réfléchir sur le concept de point de vue revient à exercer une sorte de retour sur l'expérience narratologique. En narratologie, le point de vue est à l'honneur grâce à une longue tradition motivée par la recherche des critères fiables dans l'approche scientifique des textes littéraires. À première vue, on peut attribuer la notion générale de perspective aux liens qui s'établissent entre histoire et récit ou récit et discours (Genette 1972). D'après Linvelt (1989), un autre courant se l'approprie afin de pouvoir penser l'approche globale des textes en termes de typologie des types textuels. Il nous semble que l'introduction de la notion de perspective a été nécessaire pour miner le caractère tout puissant du narrateur. L'accès à l'histoire est relatif, et sa relativité peut être exprimée dans trois sens spécifiques. La focalisation zéro, la focalisation extérieure et la focalisation intérieure ouvrent un nouvel horizon aux analyses en études littéraires.

Nous rappelons que la perspective est une façon de gérer les informations. L'organisation des informations dépend de la perception du sujet percevant, et elle en est ainsi restreinte. Que ce soit le narrateur, un des personnages, ou les deux, par leur statut de sujet percevant, ils servent de filtre pour éliminer certaines des informations et en retenir d'autres qui attendent d'être livrées au lecteur, et ceci, dans l'objectif de construire et de faire construire l'univers diégétique.

Dans son acception linguistique, (Banfield 1979, 1995) il est également souligné le fait que ce sujet, percevant ce qui lui est extérieur, est aussi un sujet pensant et parlant. Cependant, si c'est le cas, l'accent se met de la perspective et de l'origine de l'information à la voix pour nous faire entrevoir un moment où le point de vue n'est pas une question de regard. Ainsi, la voix semble plus adéquate pour rendre compte de ce qui s'exprime réellement dans le texte. Ce principe s'apparente par ailleurs aux principes fondateurs de la théorie de la polyphonie. Nous avons l'intention d'y revenir plus loin, ici, nous nous bornerons à mettre en évidence une autre tendance linguistique qui, pour le concept de 
point de vue, utilise le terme empathie indiquant le déplacement mental de sa propre place à celle de l'autre. Plus exactement, cette catégorie grammaticale renvoie au domaine de marquages syntaxiques pour rendre compte de l'intersubjectivité, à savoir de l'adoption, de la part du locuteur, d'un point de vue d'un participant au procès. La théorie de l'empathie (Kuno et Kaburaki 1977) a introduit par ailleurs le concept de camera angle, lieu de représentation syntaxique et morphologique du fait qu'un même contenu propositionnel peut être représenté de différentes manières. En fonction de la distance du locuteur par rapport aux participants rendus dans le contenu propositionnel - à savoir en fonction de son empathie vis-à-vis d'eux - il a deux possibilités à représenter la situation dont il parle en l'affectant de marquage : ou bien il prend la camera à une distance égale ou il la prend plus proche de l'un que de l'autre. Le mouvement de la caméra se réfléchissant dans la syntaxe peut être illustré avec les phrases de (1) à (3) : dans (1) on peut observer une même distance vis-à-vis de Pierre et de Marie, dans (2) c'est Pierre qui est affecté de l'empathie du locuteur et dans (3) c'est Marie.

(1) Pierre embrasse Marie.

(2) Pierre embrasse son amoureuse.

(3) Marie est embrassée par son amoureux.

21 À bien y réfléchir, selon cette conception, le concept de point de vue - certainement à cause de son utilisation métaphorique - recouvre le concept de thème ${ }^{8}$ (topic), dans la mesure où les deux concourent à gérer les informations véhiculées par les phrases; ils continuent à être étroitement liés à la perception. Car, même si on envisage la double dimension de l'empathie, posture compréhensive et posture relationnelle, renvoyer à la capacité du locuteur de reconstruire ce que l'interlocuteur a vu ou pensé, voire de le partager, ne permet pas de renvoyer directement à la capacité de parler à sa place. De ce fait, il sort du cadre linguistique proprement dit.

Ce qui est commun dans l'usage des termes perspective, focalisation, empathie ou camera angle, c'est qu'ils présupposent, sans exception, que l'information dans le texte ou dans la phrase a son ancrage qui est déterminé, explicitement ou implicitement, par la perception. Il est frappant de constater que l'ancrage du concept dans une phénoménologie se retrouve également chez la plupart des auteurs qui utilisent le terme point de vue.

\subsection{Focalisation : narratologie et neurosciences}

On ne saurait ignorer le domaine des neurosciences dans lequel le point de vue est un outil pour décrire la mémoire. Si nous nous permettons de faire juste une incursion éclair dans un champ disciplinaire relativement jeune, c'est pour laisser entrevoir que, malgré l'usage particulier du concept de point de vue, il reste assimilé à la perception. Dans le travail dont l'objet est la mémoire épisodique de Bergouignan (2009), le point de vue est relatif à l'imagerie mentale. L'individu visualise ses expériences du passé, et cette visualisation n'est pas toujours donnée dans la perspective originelle. Si le terme de point de vue correspond à la perspective associée au souvenir, pour le rappeler, il y a deux possibilités : d'une part, renvoyer la scène en point de vue Acteur, le revoir comme à l'époque à la première personne, focalisation sur le contexte environnant, avec l'effet d'enregister les événements ; d'autre part, renvoyer la scène en point de vue Spectateur, la revoir à la troisième personne, focalisation sur soi, comme si on était filmé (Nigro et Neisser 1983), avec l'effet d'analyser les événements. Nous ne sommes pas dans la position de prendre la mesure de l'intérêt de cette distinction qui consiste certainement à faire 
voir les conséquences du changement de point de vue dans un souvenir sur la perception de soi, et par extension, ouvre une nouvelle voie dans la compréhension des troubles et déficit de la mémoire. Pour notre propos, ce qui est à noter, c'est que, dans les deux cas de figure, le point de vue n'est pas détaché de la perception sur la scène passée.

\subsection{Point de vue : narratologie et linguistique}

Nous passons maintenant, parmi les définitions qui s'affichent comme linguistiques utilisant le terme même de point de vue, à celle qui se donne pour objectif d'étendre le concept de «la perspective narrative» (Rabatel 1997 : 287), - par ailleurs synomyme de focalisation - de telle manière qu'il inclut au delà de la perception «la perception représentée » (Rabatel 1998: 54) indiquant les processus mentaux. Cet élargissement aboutit à réduire le nombre des focalisations à deux : ainsi, on travaille avec le point de vue du narrateur et avec le point de vue du personnage. Une des préoccupations de Rabatel est de porter un jugement critique sur des analyses traditionnelles qui ne font rien d'autre que d'identifier le sujet comme porteur de point de vue. Limitées à répondre aux questions Qui voit? Qui sait?, ces analyses ne font que nommer le focalisateur à savoir l'instance qui occupe la position à partir de laquelle les choses peuvent être vues et connues ou le foyer d'où provient un événement. Malgré ce regard critique, nous trouvons que pour que cette réduction puisse être effectuée, il lui est nécessaire de tenir à la thèse suivant laquelle le point de vue - que ce soit celui du narrateur ou du personnage, puisqu'ils ont le même statut - doit être lié à un sujet.

Une autre remarque critique de Rabatel concerne le caractère métaphorique du point de vue : selon lui, il ne pourra être enlevé tant qu'il ne soit pas linguistiquement fondé. Nous ne pouvons que saluer l'idée de reconnaître cette nécessité. Cependant, quoi entendre exactement par linguistiquement fondé ? Ce qu'il propose, c'est de « rechercher les traces linguistiques d'un PDV» (Rabatel 1997: 287). Suivant cet objectif, parmi ces traces linguistiques nous retrouvons entre autres: 1 . aspectualisation, à savoir l'ensemble des commentaires concernant les propriétés de l'objet perçu; 2. les plans secondaires textuels (en opposition avec les plans primaires) en tant que lieux des point de vue construits ; 3. l'imparfait ; 4. les anaphores associatives (Rabatel $1998: 54$ ).

Il nous parait clair que le terme de point de vue est destiné à relier les perceptions et les processus mentaux dont une des expressions possibles est le présentatif c'est disposant d'une valeur énonciative (Rabatel 2000). Reprenons un peu les perceptions. Si les informations sont communiquées directement sur la base de la perception, elle apparaît sur le plan de la phrase comme une évidentialité en nous révélant aussi les sources de ces informations, sans constituer pour autant des connaissances. Dans d'autres cas, sur la base de la perception représentée, la sélection des informations dépend déjà d'un point de vue, relatif au narrateur ou au personnage, ce qui n'est saisissable que sur le plan du texte 9 .

Notre remarque critique vise précisément l'impossibilité de discerner ce qui relève de la perception et du savoir. Leur indiscernabilité est due d'une part à l'interprétation, d'autre part, à la pensée représentée. Si la perception représentée inclut la pensée représentée, comment isoler l'information ainsi véhiculée du savoir? Sans parler du fait que le problème de l'isolation, parce que la perception est relative au savoir, nous reconduit aux questions du départ Qui voit? Qui sait?, questions que Rabatel a rejetées comme non pertinentes. 
28 Notre autre remarque critique concerne l'extension du terme de point de vue chez Rabatel. Il recouvre tout phénomène qui est relatif à l'expression linguistique de la perception. Selon nos considérations, l'exploration de l'expression linguistique de la perception ne nous amène pas automatiquement au fondement linguistique $\mathrm{du}$ concept de point de vue. Ce qu'on fait, c'est de mettre en évidence les éléments du discours qui renvoient à un énonciateur à la troisième personne qui n'est pas identique au sujet parlant. Puisque cette instance est responsable fondamentalement des perceptions et des pensées indépendamment de toute activité de l'énonciation, elle doit présupposer un sujet de conscience (Rabatel 2000). De surcroît, parler des contextes du discours dans l'objectif de reconstruire la situation d'énonciation ne va pas garantir non plus le fondement linguistique du point de vue. À nos yeux, à l'origine de cette tension, c'est l'assimilation du discours à la langue. Une des raisons en est certainement le caractère relatif du point de vue à la référenciation qui nous renvoie tôt ou tard à une correspondance entre discours et situation d'énonciation.

Cette confusion ne disparaîtra pas non plus au moment où il devient évident que le point de vue n'est pas un contenu, mais il est une opération. Grâce à son statut d'opération, le point de vue fait apparaître le sujet énonciateur indissociable du monde des référents dans une même unité indivisible. Le fait de dire inclut aussi la référenciation, la manière dont le locuteur renvoie aux objets du monde. Les différents modes dont le locuteur renvoie aux objets du discours tels que dénomination, catégorisation, modalités, trahissent des choses du sujet (Rabatel 2005).

30 Nous pouvons remarquer un décalage de la perspective et des processus mentaux vers la référenciation tout en restant dans le cadre du discours. Cependant, il ne suffit pas d'accepter que les modes de référenciation caractérisent essentiellement le locuteur pour dire du nouveau ; avec cela, on ne fait qu'accepter que le sujet modal pourrait s'exprimer en dehors des attitudes propositionnelles de manière implicite, laissant des traces dans le dictum. Nous citons :

En réalité, le PDV ne réside pas dans un contenu (par exemple des perceptions, des paroles ou des pensées), il réside dans la manière dont tous les objets du discours sont référenciés en fonction d'une subjectivité, de telle façon que les choix de référenciation - sélection des référents, organisation de ces derniers dans le cadre discursif, dénomination, désignation, qualification, modalités et modalisations, etc. - renseignent sur l'objet et sur le sujet énonciatif à l'origine de la référenciation. En définissant le PDV par des opérations linnguistiques, plutôt que par des contenus, on entend signifier que les mécanismes linguistiques de l'énonciation et de la référenciation sont solidaires, à l'instar du recto et du verso d'une feuille de papier.[...] marques qui relèvent du sujet modal, lesquelles s'expriment non seulement dans le modus, à travers les réactions du sujet à l'égard des contenus propositionnels, mais aussi dans le dictum lui-même, en sorte que l'énonciation englobe la manière dont les objets sont référés, par rapport au locuteur. (Rabatel $2005: 231)$

31 Suivant nos remarques critiques, pour notre propos, il ne sera pas pertinent de distinguer un point de vue narratif et un point de vue linguistique. Nous allons les réunir sous l'étiquette de théories des traces, ainsi toute tentative qui présuppose une source d'informations ou un ensemble de renseignements sur la perception. Le concept de point de vue sera ainsi défini en termes de sujet percevant, pensant, parlant même si seulement d'une manière indirecte à travers des modes de référenciation. Le point de vue devient une entité non linguistique, car la sémantisation de l'information dépendrait de son 
traitement par le système de représentation perceptive, et de l'aptitude du sujet à changer de référentiel.

La question s'impose: Est-il concevable de poser un concept de point de vue indépendamment de tout sujet pensant? Si oui, en quoi serait-il efficace ou plus puissant sans la prise en compte de ce dernier? Sous quelles conditions peut-on dire que la subjectivité attachée non plus au discours mais à la langue soit en même temps détachée de la perception aussi bien que de la situation d'énonciation et, comme composante essentielle, de la signification sans être réduite pour autant à la référenciation?

\section{Théorie des topoi : une alternative à la théorie des traces}

Admettons que dans le dit, il y a le dire ; l'énoncé porte en soi les traces de l'énonciateur. En effet, il est rationnel de croire que suivant ces traces, il est possible d'identifier l'énonciateur en sélectionnant un sous-ensemble d'éléments linguistiques qui sont à notre disposition, notamment embrayeurs, pronoms personnels, modificateurs, intensifieurs, adjectifs évaluatifs, entre autres.

Qu'est-ce que cette concession implique sur le concept de point de vue ? Il sera destiné à recouvrir un sous-ensemble d'éléments assez restreint. D'une part, il y a des éléments linguistiques qui renvoient directement à l'énonciation et il y en a certains qui le font seulement de manière indirecte, surtout si nous tenons compte aussi des possibilités d'ordre axiologique, culturel ou idéologique. Ainsi, la définition linguistique du point de vue revient à dresser une liste exhaustive.

À nos yeux, le concept de point de vue devient pertinent pour les études linguistiques à partir du moment où on prend en compte le démembrement du sujet parlant et le triomphe de la connotation sur la dénotation. Rappelons dans ce qui suit les deux moments décisifs que nous pouvons considérer comme antérieurs de la sémantique des points de vue. En dehors de la connotation, c'est la polyphonie qui est considérée comme signe précurseur dans la mesure où elles conduisent à formuler la nécessité d'introduire une nouvelle notion, celle de topos.

\subsection{Connotation}

Pour observer le phénomène de connotation, nous rappelons la liste des possibilités qu'elle recèle, établie par Hjlemslev ([1943] 1968); si les mots sont dotés en dehors de leur signification primaire d'autres significations secondaires, c'est parce que la langue est l'expression dont le contenu est déterminé par la culture. Cependant, il n'est pas nécessaire d'articuler le signifiant et le signifié, selon l'expression et le contenu, car, comme Ducrot le déclare, le signifiant, à savoir « [...] le fait même que ce qu'on dit est un énoncé linguistique» (Ducrot 1966: 43). Avant d'évoquer un certain contenu d'ordre culturel, il faut prendre en compte le fait de dire dans le dit.

Cette observation permet de fonder non pas la théorie des traces, mais la thèse fondamentale, selon laquelle la signification est d'ordre argumentatif. Dans la théorie de l'argumentation, tout énoncé n'est pas fait pour argumenter, mais la phrase, même sous sa forme interrogative, est susceptible d'être utilisée dans une argumentation. L'orientation argumentative permet de traiter ensemble langue et discours. Si on admet 
que tout énoncé peut être considéré comme argument en faveur d'une conclusion, ce qui est en jeu c'est de ùettre en évidence ce qui garantit ce lien.

Mais reprenons un instant la connotation dont Kerbrat-Orecchioni (1977) a fait une synthèse. Pour certains, elle signifie la force évocatrice des mots porteurs d'une valeur émotionnelle, alors que pour d'autres, au contraire, elle rassemble les déterminations culturelles et sociales sans parler de ceux qui n'en voient qu'un sous-ensemble de traits du concept ou du signifié.

Par exemple, le mot chien suivant sa dénotation désigne un groupe d'animaux et, en fonction de la communauté linguistique, évoque l'idée de la fidélité ou la conduite abjecte, tout comme l'hexagone dénotant la France évoque l'idée de l'équilibre ou celle du centre. À propos de ces connotations, on ne dirait pas qu'elles soient individuelles. Par définition, «[...] la connotation signifie ici l'association stable d'une signification dérivée, secondaire avec la signification de base » (Todorov $1967: 29$ ).

40 Ce qui retient dans cette définition notre attention, malgré le fait qu'il n'y ait aucune allusion à l'énonciation, c'est l'usage de «stable ", puisqu'à partir du moment où les connotations non individuelles mais relatives à une communauté sont qualifiées de stables, sans être néanmoins universelles, on n'est pas très loin d'en reconnaître une des propriétés essentielles du topos, à savoir celle de l'intersubjectivité : être présenté comme partagé. C'est cette propriété stable qui rend le topos susceptible de devenir le critère de l'observabilité au sein du modèle sémantique. Cependant, il faut aussi admettre que la langue est un ensemble d'instructions et non pas le trésor des sens dénotationnel et connotationnel. Barthes y contribue largement, même s'il n'en reconnaît qu'assez tardivement la déchéance hiérarchique. Il parle d'abord en termes de sens seconds :

La société développe sans cesse, à partir du système premier que lui fournit le langage, des systèmes de sens seconds et cette élaboration, tantôt affichée, tantôt masquée, rationalisée, touche de très près à une véritable anthropologie historique. (Barthes 1964: 80)

41 Quelques années plus tard, il lui devient clair (Barthes 1970) que la primauté de la dénotation sur les connotations est illusoire. Il laisse tomber la dénotation pour ne plus parler qu'en termes de connotations : la dernière dans la série d'associations sert à mettre fin à l'interprétation et à la lecture.

42 Sur cette base, il est assuré que les significations d'un seul mot ne se classent pas comme primaires et secondaires : en effet, il serait absurde de dire que certaines instructions sont primaires ou essentielles alors que d'autres secondaires ou accidentelles. Nous trouvons que la notion de connotation a été peu exploitée malgré les élans donnés par Barthes et Todorov et elle n'a pas pu être réellement comprise et réinvestie dans le domaine de la linguistique avant l'apparition de la version topique de la théorie de l'argumentation dans la langue.

43 Ce n'est pas étonnant: c'est la première fois qu'une sémantique qui se passe de l'information / dénotation / référence se met au programme à long terme. C'est ainsi que la notion de topos l'emporte sur celle de connotation dans les travaux de Raccah (Bruxelles, Ducrot et Raccah 1995 ; Raccah 1998). Les topoi en tant qu'indicateurs des usages propres à une communauté linguistique ne seront pas secondaires par rapport au système de la langue. Au contraire, ils seront susceptibles de rendre compte de ce qui $y$ est essentiel, reléguant ainsi au second plan la dénotation, la référence ou la référenciation. Ainsi, la question de savoir comment faire correspondre à une entité non linguistique une séquence linguistique perd toute sa pertinence. 


\subsection{L'éclatement du sujet}

Suivant la précédente piste intéressée à la nature du sens, on aboutit tôt ou tard à la reconnaissance de l'inutilité de la dénotation. Mais qu'en est-il avec la représentation? Avec les états mentaux du sujet parlant? Si le sujet peut ne pas prendre en charge ce qu'il dit ou ce qu'il fait, du coup, il est affecté dans son intégrité. Quoi faire face à l'hétérogénéité qui en découle, et qui se manifeste à travers les multiples attitudes vis-àvis de l'énonciation comme événement ou action? En effet, on insiste depuis Ducrot (1984) sur l'éclatement du sujet en une pluralité de dimensions : il est communément reconnu que le sujet se divise en sujet parlant, en locuteur et en énonciateur. Considérons-les.

Le sujet est tout d'abord une entité empirique dotée d'un ensemble de propriétés qui, indépendamment de son énonciation, permettent de le considérer dans ses rapports sociaux. Parmi ces propriétés, on lui reconnaît celle qui le désigne comme producteur de paroles. Donc, le sujet parlant comme acteur d'une société est déterminé par la situation communicationnelle dans laquelle il s'est engagé : en prenant la parole, il a aussi la chance de s'affirmer et d'afficher son identité sociale.

Ensuite, comme locuteur, il est une entité discursive qui est responsable de l'énonciation tout en restant à l'extérieur de sa propre énonciation à l'instar du sujet parlant. Il est cependant déterminé cette fois-ci par ses propres paroles et ses stratégies discursives. Nous pouvons dire qu'en dehors de son identité sociale, il dispose d'une identité discursive. Grâce à cette identité manifestée, il laisse aussi entrevoir les conditions du dire. Ce qui implique un autre strate du sujet.

Enfin, il est considéré comme énonciateur qui est en mesure d'attribuer des points de vue. Il s'agit alors d'une entité qui est incluse dans son énonciation sans la nécessité de se situer à l'extérieur. Cette entité diffère donc des deux premières dans la mesure où elle se caractérise sur la base des croyances, des connaissances acquises ou supposées. Ducrot définit les énonciateurs comme des " êtres [...] censés s'exprimer à travers l'énonciation, sans que pour autant on leur attribue des mots précis ; s'ils « parlent », c'est seulement en ce sens que l'énonciation est vue comme exprimant leur point de vue, leur position, leur attitude, mais non pas, au sens matériel du terme, leurs paroles » (1984: 204).

Cette articulation tripartie suggère de reconsidérer quatre points :

- Premièrement, celui qui produit l'énoncé, s'il en est responsable, il n'est pas forcément respnsable de l'énonciation même. Ce qui permet de voir l'énonciation séparée de celui qui l'a effectuée.

- Deuxièmement, ce n'est pas la subjectivité du sujet parlant qui fonde la langue. Le sujet se constitue dans l'énonciation. Cette subjectivité dans la langue (Benveniste 1966 : 262) ne signifie pas le caractère subjectif de la parole du sujet, elle est singulière certes. Mais cette subjectivité propre à la langue s'exprime indépendamment de la prise en compte de l'énonciation, de l'usage. Comment rendre explicite cette subjectivité ; c'est ce qui est en jeu d'abord dans la théorie de l'argumentation proposée par Anscombre et Ducot, ensuite dans la théorie des topoi, et enfin dans la sémantique des points de vue conçue par Raccah.

- Troisièmement, les caractérisations précédentes manquent de rigueur, mais elles sont nécessaires pour pouvoir indiquer par rapport à quels éléments peut être pertinent le concept linguistique du point de vue.

- Quatrièmement, les caractérisations précédentes sont facilement conciliables avec notre intuition suivant laquelle certains énoncés laissent entendre une voix 
qui diffère de celle qui parle, ce sont souvent les proverbes ou locutions proverbiales. Sinon, comment pourrait-on les comparer?

\subsection{Polyphonie}

49 L'idée de la polyphonie ${ }^{10}$ s'installe, à partir des années 80 , au cœur des réflexions d'ordre linguistique sur la nature de la langue et de la signification, dans la lignée des travaux de Ducrot. Elle sert à remettre en question l'intégrité du sujet, et surtout, à distinguer phrase et énoncé, signification et sens. On parle de polyphonie, si, au sein d'un énoncé, plusieurs énonciateurs apparaissent (Ducrot 1984: 204). Dans le prolongement de nos idées développées, il convient de souligner que ces énonciateurs, s'ils prennent des points de vue , positions, attitudes, ce n'est pas parce qu'ils seraient réductibles à la perception ou à la situation d'énonciation ${ }^{11}$. C'est parce qu'ils sont des entités discursives. Rappelons ici la négation et les subordonnées concessives pour une lecture polyphonique.

(4) Pierre n'est pas timide.

L'approche polyphonique de l'énoncé consiste à mettre en scène, au lieu de deux contenus propositionnels, deux attitudes, positions ou points de vue. En éléminant l'idée du contenu propositionnel, le propre d'un énoncé n'est pas la description d'une situation mais une position pour ou contre. Les positions peuvent être à leur tour distinguées suivant Ducrot (1993: 128) en trois catégories: évaluative comme (4), interprétative comme (5) et objective comme (6).

(5) Ce mur n'est pas blanc.

(6) Le chat est sur le paillasson.

51 Au lieu d'interroger ces ordres différents ou les critères sur lesquels cette classification est basée, ce qui nous importe ici c'est d'une part, l'abandon de la proposition avec ses valeurs de vérité, d'autre part, la prise en compte de plusieurs positions simultanées. Celui qui dit position, ne dit pas forcément opinion ou jugement assumé par le locuteur. Pourquoi ? La définition n'en contient aucun point de repère. Cependant, nous pouvons faire l'hypothèse que position / attitude / point de vue sont propres à un énonciateur d'ordre discursif, alors que opinion / jugement sont relatifs à un contenu propositionnel indépendamment de toute entité discursive. Dans ce deuxième cas de figure, nous sommes dans la distinction dictum-modus héritée de Bally (1965) : un sujet conscient prend une attitude vis-à-vis d'un contenu propositionnel, ou d'une représentation. Or, en effaçant la frontière, on doit abandonner la conception selon laquelle il serait possible de déterminer une prééminence des faits par rapport auxquels le sujet se positionne ou agit. Ce qui paraît plus adéquat c'est d'insister, à la place de cela, à donner place aux possibilités des points de vue (Ducrot 1989 : 190-191).

La polyphonie des énoncés peut être illustrée par les subordonnées concessives introduites par les connecteurs bien que, quoique, malgré que. Elles présupposent un on dit comme dans (7) suivant lequel une causalité est affirmée et que le locuteur ne prend pas en charge.

(7) Bien que Marie ait passé des années en France, elle ne parle pas le français.

Un énonciateur affirme que durant un long séjour, on apprend aussi la langue du pays en question. Mais quel est le gain de la notion de polyphonie par rapport à celle de la modalité ? Considérons d'abord les énoncés de (8) à (10) et ensuite de (11) à (12). 
(8) Pierre est doué en musique.

(9) Marie trouve que Pierre est doué en musique.

(10) Marie a tort de penser que Pierre est doué en musique. exemples de (11) à (12) avec il paraît et avec le conditionnel épistémique ${ }^{12}$, on peut
distinguer les positions : le locuteur comme auteur de l'énoncé n'adopte pas le point de
vue «Pierre est doué en musique ». On n'a accès à la représentation qu'à travers un énonciateur, même si ce dernier n'est pas identifiable.

(11) Pierre est doué en musique, parait-il.

(10) Pierre serait doué en musique.

Après avoir illustré l'intérêt de la distinction entre Locuteur et énonciateur, nous pouvons conclure provisoirement ceci : alors que le point de vue / perspective de la narratologie n'est pas formulable sans faire intervenir la perception ou la représentation du sujet, la polyphonie, précurseur du point de vue linguistique ne nécessite pas la prise en compte du sujet, et par conséquent celle de la représentation. Affirmer le caractère polyphonique de l'entité discursive revient à affirmer aussi qu'elle peut mettre en scène des énonciateurs par d'autres moyens que le discours rapporté. Le locuteur, au cœur de son dit, à l'intérieur de son énoncé fait apparaître des énonciateurs distincts de lui-même. Relatif au point de vue, cela signifie que la présence de l'autre devient évidence non seulement à travers ces paroles citées mais aussi à travers ses visions, vues et idéologies qui ne sont pas pour autant réductibles aux informations filtrées, à la perception directe ou à la connaissance incomplète. La question de savoir si un élément linguistique du système porte la trace d'un point de vue ou pas perd tout son intérêt. En posant que tout contenu sémantique est a priori une position par le simple fait de son énonciation, ce n'est pas pertinent d'aller à la recherche des éléments spécifiques qui en soient responsables, ni des sujets dont elle dépend.

Nous avons vu que la polyphonie était nécessaire pour justifier l'éclatement du sujet intègre; or les positions issues des hétérogénéités coincident forcément avec les opinions. Leur distinction n'a pas pu être fondée sur une entité discursive, non empirique disposée à être multiple. Intuitivement, nous saisissons cette nuance à l'analogie de celle qui s'établit entre les voix qui pénètrent à travers les fenêtres fermées d'une chambre. Certaines voix laissent reconnaître leur source (correspondance avec position), alors que d'autres, au contraire, ne laissent pas la révéler (correspondance avec opinion). À partir de là, des travaux se développent pour rentabiliser cette conception polyphonique sur le 
plan du discours. Haillet (2007) décrit les représentations discursives des objets, propriétés ou événements en termes de points de vue, de préférence à l'énonciateur.

\section{Point de vue en termes de champ topique} il convient de réfléchir à la question de savoir ce qui lui substitue ${ }^{13}$. Nous avons vue que l'énoncé ne perd pas son caractère subjectif en éliminant la pensée et les représentations du locuteur. Ce qui remplace en même temps les deux compartiments isolés, dictum et modus c'est le point de vue. Pour éviter la multiplication des points de vue et par conséquent celle des sources de l'information, il est nécessaire de recourir à la notion de topos, sur la base duquel le fait que nos discours sont orientés peut être justifié. Nos énoncés ne renvoient pas directement au monde, mais au discours. Entre le sujet et le monde, ce sont les voix, ou les principes argumentatifs qui font l'intermédiaire. Le sens se construit sur la base des ces discours dont on peut rendre compte avec les topoi. Or, ces principes argumentatifs ne caractérisent pas seulement les discours mais sous-tendent aussi la langue. L'argumentation d'un discours ne recoupe pas nécessairement l'intention communicative, même si nous savons que certains discours dont tout particulièrement la polémique manifeste l'intention d'argumenter. Si nous admettons que l'argumentation ne peut pas être donnée en termes d'intention, alors elle doit être propre de la langue et $a$ fortiori des phrases et des mots. Ainsi, l'argumentation n'est pas disposition mais contrainte. Définir le topos comme $\pm \mathrm{P}+\mathrm{Q}$ ne dit rien à propos de $\mathrm{P}$, ni de $\mathrm{Q}$, même si la correlation est évidente aussi bien que leur position dans une graduation. Ce qui reste à expliciter, c'est la détermination sémantique de cette correlation, en termes de champs topiques (Raccah $2002: 263-264$ ).

\subsection{Signification, contrainte et présupposition}

61 Désormais, dans la description lexicale - depuis les travaux de Bruxelles et Raccah (1992: 66) - les champs topiques cristallisés sont susceptibles de rendre compte des connotations 
habituelles mais aussi des contraintes qui pèsent sur les topoi évoqués par les énoncés. Les points de vue suggérés ou imposés, résultats de la description linguistique sur le plan du discours aussi bien que sur le plan de la langue, permettent de gérer ce qui dépend de la situation et ce qui en est indépendant.

Ces contraintes exercées par les articulateurs et les mots du lexique sont amplement étudiées ailleurs (Várkonyi, Simonffy,Tordesillas) dans ce numéro de la revue. Ici, nous proposons de considérer un point qui en est resté à l'ombre. Le topos a été introduit d'abord pour qualifier la relation entre argument et conclusion, et la présupposition, pour Ducrot, prend une place primordiale dans la spécification des rapports que les topoi et les enchaînements discursifs entretiennent entre eux. Or, dans le cadre de la Sémantique des points de vue, certains aspects de la présupposition sont aussi à réviser. Pour caractériser de manière adéquate le sens des énoncés, la nécessité de la prise en compte du point de vue apparaît dans les phases préparatoires du modèle: c'est une condition qui remplace les conditions de vérité des propositions. Vérité ou point de vue, les deux ont un caractère contraignant, certes, sauf qu'ils n'ont pas le même statut, ce qui s'explique par les différentes visions sur la présupposition. Dans le sens classique du terme, elle est relative aux faits exprimés par des propositions, alors que dans le sens argumentatif du terme, elle devient relative aux points de vue. Dans ce dernier cas de figure, même les conditions d'emploi restent non pertinentes, car les conditions d'énonciation concernent les enchaînements discursifs des énoncés et non pas l'assertabilité des propositions (Raccah $2002: 252$ ).

Les contraintes, visant à déterminer la signification dans la construction du sens ou dans l'interprétation, interviennent en tant que présuppositions afin de spéficier ce qui se mettrait autrement dans la case passe-partout des connaissances préalables ou d'arrièrefond.

\section{Conclusion}

En somme, dans notre aperçu sur les usages du concept de point de vue, nous avons argumenté en faveur d'une distinction essentielle, celle qui est mise en évidence par l'opposition marquage vs contrainte. La conception fondée sur les contraintes, en opposition avec celle qui opte pour les points de vue marqués, ne semble pas compatible fondamentalement à cause de la présupposition précédemment définie, - avec la conception fondée sur les traces. Le point de vue en termes de champ topique n'est pas le privilège d'un sous-ensemble d'éléments, mais une propriété essentielle sémantique de chacun des éléments linguistiques.

Le point de vue est considéré ou bien comme une entité extralinguistique, mais qui peut être linguistiquement marquée, ou bien, au contraire, comme propriété inhérente à la langue contribuant ainsi à trahir les idéologies des locuteurs, à donner accès à quelque chose d'extralinguistique. Dans le premier cas, les points de vue résultent de la perception limitée, ainsi cette conception ne sort pas du cadre que le point de vue comme catégorie narratologique nous livre. Dans l'autre cas, les points de vue, par leur statut de présupposition, ne sont pas réduits à un ensemble limité d'éléments.

Le point de vue étant une des propriétés objectives des mots-de-langues, indépendante de nos capacités de concevoir ou d'imaginer, permet de rendre compte de la structure de la signification sans en nécessiter la prise en compte d'une stratification ou 
d'hiérarchisation quelconque. Le fait qu'il y ait ou non un sujet de conscience qui aurait choisi tel ou tel point de vue n'entre pas en ligne de compte. Cela nous semble rejoindre, dans une autre perspective et un autre raisonnement, l'idée, avec l'expression de Putnam, que « les significations ne sont pas dans la tête ». Ainsi, il est possible d'utiliser un mot dans son discours, sans savoir ce que le mot désigne, sans savoir ce dont il est vrai. La connaissance de la signification revient à connaître un ensemble de contraintes qui, elles, ne dépendent pas de la nature du monde actuel non plus.

Dans la plupart des cas, les usages ne font pas apparaître le concept de point de vue mais seulement le concept de ce qui joue le rôle de point de vue. La Sémantique des Points de Vue permet de passer de ce qui est concevable à ce qui est nécessaire, même si difficilement concevable, à savoir inclure dans la description sémantique le point de vue. Notre système cognitif ouvre un grand nombre de possibilités d'interprétation, seulement le système linguistique contraint la construction du sens, résultat de l'inteprétation. Cependant, cela ne signifie pas en soi une limitation, puisque les contraintes concernent la manière dont une interprétation résulte du sens sans limiter pour autant le nombre de possibilités d'appréhender une situation en particulier et le monde en général.

Pierre-Yves Raccah a choisi de suivre un parcours qui montre comment langue et discours se rejoignent sans jamais s'identifier l'un à l'autre tout en ouvrant de nouvelles pistes devant les considérations linguistiques, culturelles et cognitives.

\section{BIBLIOGRAPHIE}

Aumont Jacques, L'image, Paris, Nathan, 1990.

Aumont Jacques, « Le point de vue », Communications n 38, 1983, pp. 3-29.

Bally Charles, Linguistique générale et linguistique française, Bern, Francke, 1965.

Barthes Roland, Éléments de sémiologie, Paris, Seuil, 1964.

Barthes Roland, S/Z, Paris, Seuil, 1970.

Barthes Roland, « Préface », in Flahault François, La parole intermédiaire, Paris, Seuil, pp. 7-10, 1978.

Boillat Alain, « Le point de vue », in Gervereau Laurent (ed.), Dictionnaire mondial des images, Paris, Éditions Nouveau Monde, 2006, pp. 834-837.

Banfield Anne, « Où l'épistémologie, le style et la grammaire rencontrent l'histoire littéraire : le développement de la parole et de la pensée représentée », Langages nº 49, 1979, pp. 9-26.

Banfield Anne, Phrases sans parole, Paris, Seuil, 1995.

Benveniste Émile, Problèmes de linguistique générale, Paris, Gallimard, 1966.

Bergouignan Loretxu, Rappel épisodique en mémoire autobiographique et point de vue : études comportementales et de neuroimagerie. Neurons and Cognition, Thèse de doctorat, Université Pierre et Marie Curie-Paris VI, 2009. [En ligne : https://tel.archives-ouvertes.fr/file/index/docid/431166/ filename/2009_Bergouignan.pdf. 
Bruxelles Sylvie et Raccah Pierre-Yves, «Argumentation et sémantique : le parti-pris du lexique », in De Mulder Walter, Franc Schuerewegen et Liliane Tasmowski (eds), Énonciation et Parti-pris, Amsterdam-Atlanta, Rodopi, 1992, pp. 59-73.

Bruxelles Sylvie, Ducrot Oswald et Raccah Pierre-Yves, « Argumentation and the lexical topical fields », Journal of Pragmatics vol. 24, nos 1-2, 1995, pp. 99-104.

Calvet Louis-Jean, Essais de linguistique. La langue est-elle une invention des linguistes ?, Paris, Plon, 2004.

Dendale Patrick et Coltier Danielle, «Point de vue et évidentialité ", Cahiers de praxématique $\mathrm{n}^{\circ} 41$, 2003, pp. 105-130.

Ducrot Oswald, « Le roi de France est sage : implication logique et présupposition linguistique », Études de linguistique appliquée, 1966, pp. 39-47.

Ducrot Oswald, Le Dire et le dit, Paris, Éditions de Minuit, 1984.

Ducrot Oswald et alii, Les mots du discours, Paris, Minuit, 1980.

Genette Gérard, Figures III, Paris, Seuil, 1972.

Hjelmslev Louis, Prolégomènes à une théorie du langage, Paris, Minuit, [1943] 1968.

Keerbrat-Orecchioni Catherine, La connotation, Lyon, Presses Universitaires de Lyon, 1977.

Kuno Susumu et Kaburaki Etsuko, «Empathy and Syntax », Linguistic Inquiry vol. 8, nº 4, 1977, pp. 627-672.

Larcher Pierre, «Le concept de polyphonie dans la théorie d'Oswald Ducrot », in Vion Robert (ed.), Les sujets et leurs discours. Énonciation et interaction, Aix-en-Provence, Publications de l'Université de Provence, 1998, pp. 203-224.

Linvelt Jaap, Essai de typologie narrative. Le point de vue, Paris, Corti, 1989.

Nigro Get et Neisser Ulrich, «Point of view in personal memories ", Cognitive Psychology vol. 15, $\mathrm{n}^{\circ}$ 4, 1983, pp. 467-482.

Haillet Pierre Patrick, Pour une linguistique des représentations discursives, Bruxelles, De BoeckDuculot, 2007.

Lévi-Strauss Claude, La Pensée sauvage, Paris, Plon, 1962.

Rabatel Alain, Une Histoire du Point de vue, Metz, Université de Metz, 1997.

Rabatel Alain, La construction textuelle des points de vue, Lausanne, Delachaux et Nietslé, 1998.

Rabatel Alain, « Valeurs représentative et énonciative du "présentatif” c'est et marquage du point de vue ", Langue française $\mathrm{n}^{\circ} 128,2000$, pp. 52-73.

Rabatel Alain, « Le point de vue, une catégorie transversale », Le Français Aujourd'hui, nº 151, 2005, pp. 57-68.

Rabatel Alain, «Perspective et point de vue », Communications vol. 85, n 1, 2009, pp. 23-35.

Raccah Pierre-Yves, «L'argumentation sans la preuve : prendre son biais dans la langue », Cognition et Interaction vol. 2, $\mathrm{n}^{\text {os }}$ 1-2, 1998, pp. 1-16.

Raccah Pierre-Yves, « Lexique et idéologie : les points de vue qui s'expriment avant qu'on ait parlé », in Carel Marion (ed.), Les Facettes du dire. Hommages à Oswald Ducrot, Paris, Kimé, 2002, pp. 241-269.

Todorov Tzvetan, Littérature et signification, Paris, Larousse, 1967. 
Voloshinov V. N., МАРКСИЗм И ФИЛОсОФИЯ ЯЗЫКА [Le marxisme et la philosophie du langage], Leningrad, Priboj, traduction française, sous le nom de Mikhaïl Bakhtine (V. N. Volochinov), Le marxisme et la philosophie du langage. Essai d'application de la méthode sociologique en linguistique, Paris, Minuit, [1929], 1977.

\section{NOTES}

1. Le point de vue, dans ce contexte épistémologique, est en lien étroit avec une théorie de la connaissance dans la mesure où connaître revient à trouver le bon point de vue.

2. À ce propos, voir une analyse approfondie dans Rabatel (2009).

3. Ce qui fait écho à la conception saussurienne suivant laquelle le point de vue constitue l'objet.

4. C'est peut-être dû au fait que le cadrage est réalisé techniquement par le cameraman.

5. Par ailleurs, la focalisation n'est pas exempte de connotations phénoménologiques qui se précisent dans la perception des phénomènes du monde sans se réduire à l'œil, à travers tous nos sens produisant une vue subjective.

6. Pour des approfondissement de la question voir Boillat (2006).

7. Souvent, les présuppositions sur la nature de la langue sont très loin d'un cadre proprement linguistique.

8. Ce serait intéressant de mener un travail dans l'objectif de creuser ces nuances; un pdv servirait à justifier alors que thème / topic viserait à déterminer la source sur la base de laquelle on pourrait justifier les traces d'un sujet dans l'énonciation narrative.

9. Voir le développement de ces rapports dans Dendale et Coltier (2003).

10. Ici, nous évitons de remonter jusqu'aux origines, ce serait un détour inutile pour notre propos. Pour une étude approfondie voir Larcher (1998). De notre part, nous signalons simplement l'importance d'une troisième piste: à travers le prisme de la problématique identitaire et de l'altérité, le caractère dialogique de tout discours (Volochinov / Baktyin : [1929] 1977) implique que le locuteur est empêché de présenter son discours de manière homogène, tout en rappelant des idéologies multiples.

11. Il convient de rappeler que dans le cadre de la théorie de la polyphonie, certains continuent à garder l'opposition dictum vs modus, et par conséquent, la notion de proposition. L'avantage est qu'il est possible de nuancer les points de vue explicitement présents tels que à mon avis, selon Pierre, et les modalités (Dendale et Coltier $2003: 111-112$ ).

12. Ducrot et al. (1980: 46) ; Ducrot (1984: 154).

13. Bien évidemment, cet abandon ne signifie pas que la langue ne puisse servir à informer.

\section{RÉSUMÉS}

Dans le cadre de notre contribution, nous nous proposons de mettre en évidence le relief que Pierre-Yves Raccah a donné au concept de point de vue, par ailleurs décisif à l'évolution interne de la sémantique. Chacun l'a utilisé dans ses variantes telles que perspective, focalisation, empathie, camera angle avant tout comme un outil de décentrement du sujet, mais c'est lui qui l'a installé, dans la perspective d'une discipline, antérieur à toute détermination cognitive ou phénoménologique. Il voit dans le point de vue un dispositif scientifique permettant une avancée 
à la fois théorique et descriptive en la recherche de possibles réponses à un grand nombre d'interrogations concernant la langue, la signification des unités linguistiques et leurs effets de sens. Cet hommage tend à mettre en valeur, entre héritage et réappropriation, son apport à la définition sémantique du concept en tenant compte de ce qui retarde et favorise l'émergence de ce concept en sémantique.

This paper aims to highlight the importance Pierre-Yves Raccah gave to the concept of point of view, it being crucial for the internal development of semantics. Everybody has used it in its various forms, such as perspective, focalization, empathy, camera angle, primarily as a tool for dismemberment of the subject, but it was Raccah who put it in place, from the viewpoint of a discipline, independently of any cognitive or phenomenological determination. For him, the point of view is a means for scientific exploration in search of possible answers to numerous questions about language, the meaning of linguistic units and their utterance meaning effect, promising theoretical and descriptive progress at the same time. This tribute aims to boost, between legacy and ownership, his contribution to the semantic definition of point of view, keeping in mind what it is that slows down and what it is that favours the emergence of this concept in semantics.

\section{INDEX}

Mots-clés : point de vue, argumentation, polyphonie, signification, contraintes, traces

Keywords : point of view, polyphony, meaning, constraints

\section{AUTEUR}

\section{ZSUZSA SIMONFFY}

Université de Pécs 